\title{
Brain metastases from an unknown primary tumour: which diagnostic procedures are indicated?
}

\author{
Marjan van de Pol, Vera C van Aalst, Jan T Wilmink, Albert Twijnstra
}

Department of

Neurology, University Hospital Maastricht,

Maastricht, The

Netherlands

$M$ van de Pol

A Twijnstra

Department of plastic and reconstructive surgery, University

Hospital Utrecht,

Utrecht, The

Netherlands

V C van Aalst

Department of

diagnostic radiology,

University Hospital

Maastricht,

Maastricht, The

Netherlands

J T Wilmink

Correspondence to:

Dr A Twijnstra, Department

of Neurology, University

Hospital, Maastricht,

PO Box 5800, $6202 \mathrm{AZ}$

Masstricht, The

.

Received 13 September 1995 and in revised form

18 March 1996

Accepted 26 March

\begin{abstract}
Seventy two patients presenting with symptomatic brain metastases from undiagnosed primary neoplasms were retrospectively reviewed. Primary malignancies were diagnosed before death in 54 patients and remained unknown in 18 patients. Lung cancer was the most common primary tumour $(72 \%)$, followed by breast cancer, colon carcinoma, and melanoma. On physical examination, 51 patients had organ specific symptoms or signs providing guidelines to the diagnostic evaluation. In 24 of the 52 patients with a primary lung tumour, and in four of the 20 patients without, organ specific complaints or findings suggested this tumour type, resulting in a positive predictive value of $85 \%$.

Overall, radiography and CT of the chest were very useful in detection of primary lung tumours. This could partly be explained by the high prior probability of detecting such tumours. Other diagnostic procedures should be used on indication only. The prognosis of patients with confirmed primary tumour position did not differ from those with unidentified primary tumour.
\end{abstract}

(F Neurol Neurosurg Psychiatry 1996;61:321-323)

Keywords: brain metastasis; unknown primary tumour site; diagnostic evaluation

In patients presenting with brain metastases from an undetermined primary tumour site, ancillary diagnostic procedures are performed to find the underlying malignancy.

A reasonable approach to unknown primary tumours would be to avoid unnecessary diagnostic procedures, without compromising the possibility of finding the primary site, weighing the overall cost of diagnostic procedures against efficacy and clinical usefulness. The focus must be on identifying a subset of patients with neoplasms that are most amenable to successful systemic treatment, such as breast carcinoma.

In $15 \%-50 \%$ of patients presenting with symptomatic brain metastases, the primary tumour remains unknown, despite extensive investigations. ${ }^{1-3}$
At the Maastricht University Hospital we retrospectively studied 72 patients presenting with symptomatic brain metastases from unknown primary tumours. The aim of the present study was to evaluate this patient group to develop guidelines for further diagnostic procedures.

\section{Patients and methods}

PATIENTS

All cancer patients referred for neurological consultation to the Maastricht University Hospital are registered. Methods used for case ascertainment have been described elsewhere. ${ }^{4}$

All cases coded as brain metastases from unknown primary cancers were included. Brain metastases had been diagnosed between 1987 and 1994, based on typical CT or MRI findings, with malignancy subsequently proved histologically. Metastases are depicted on CT or MRI as circumscribed contrast enhancing lesions with surrounding oedema and mass effect.

\section{METHODS}

Using a standard protocol, general history and physical examination data were retrospectively gathered from medical records. Information from histological examination was verified from the pathology department's database. The same procedure was followed for the radiological examinations. The radiographs were deemed abnormal if findings suggested tumour position.

The time between the first neurological consultation and the confirmation of the tumour position defines the diagnostic interval. The time from the first neurological consultation until death defines overall survival. The minimal follow up period was one year or until death.

Diagnostic intervals and survival curves were constructed using the method of Kaplan and Meier. Statistical significance was calculated by the log rank test.

\section{Results}

Between 1987 and 1994, brain metastases from unknown primary tumours were diagnosed in 72 patients, 46 men and 26 women, with a median age of 65 (range 15-85) years. 
Table 1 Organ specific complaints and findings on history and physical examination

\begin{tabular}{lcc}
\hline Localisation & $\begin{array}{l}\text { History } \\
n(\%)\end{array}$ & $\begin{array}{l}\text { Physical } \\
\text { examination } \\
n(\%)\end{array}$ \\
\hline Lung & $26(36)$ & $12(17)$ \\
Oesophagus/stomach & $12(17)$ & $2(3)$ \\
Colon/sigmoid & $8(11)$ & $1(1)$ \\
Breast & $2(3)$ & $6(8)$ \\
Melanoma & $0(0)$ & $1(1)$ \\
Kidney/urinary tract & $2(3)$ & $3(4)$ \\
Genitals & $3(4)$ & $0(0)$ \\
\hline
\end{tabular}

Brain CT was performed in 54 patients, MRI in four, and CT plus MRI in 14. Thirty eight patients had single, and 34 multiple metastases. The lesions were supratentorial in 57 , infratentorial in three, and both infratentorial and supratentorial in 12 .

Most of the patients (57\%) had complaints suggesting an underlying malignancy. Symptoms indicating primary lung cancer were most frequent. These included altered coughing pattern, hoarseness, and dyspnoea.

Physical examination disclosed abnormalities suggesting a primary tumour in 22 patients $(31 \%)$. Most of these patients had

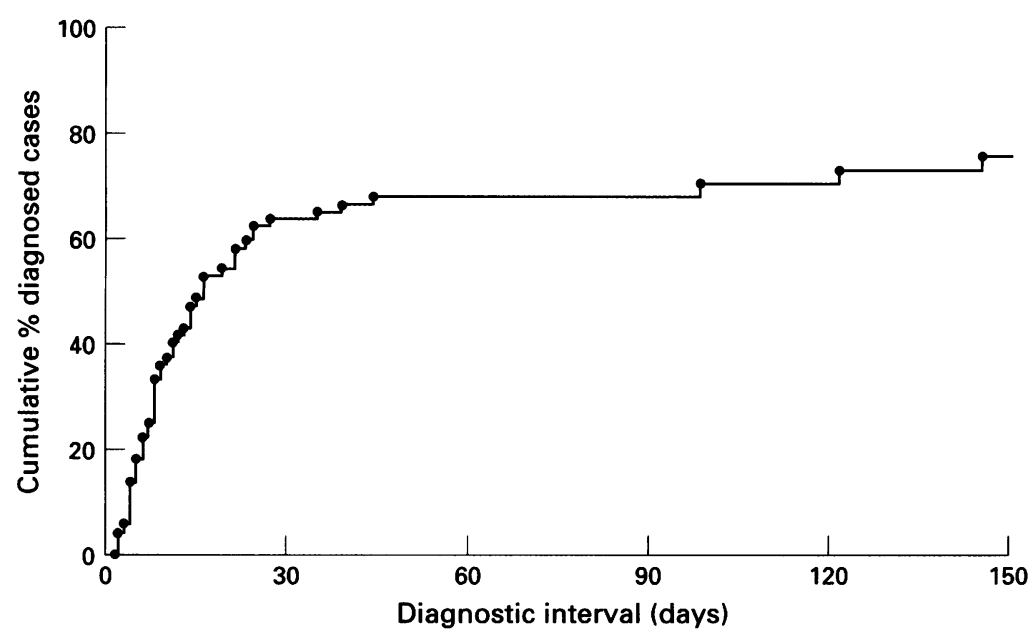

Figure 1 Diagnostic interval, time between first neurological examination and confirmation of tumour localisation. Median diagnostic interval was 15 (range 1-157) days. The curve represents the cumulative percentage of diagnosed cases.

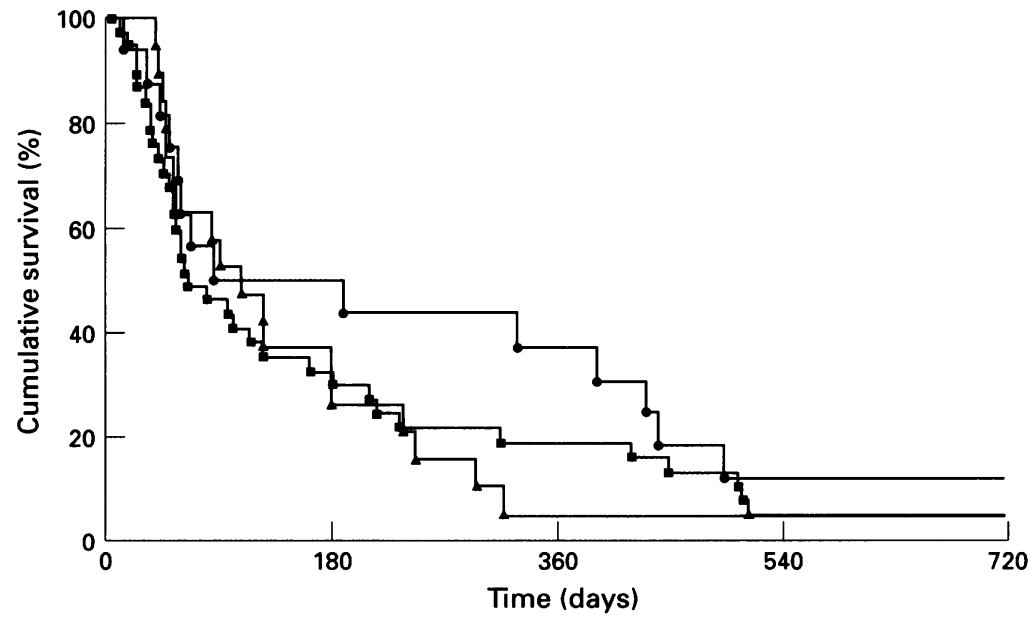

Figure 2 Survival curves of patients with a non-systemically treatable tumour ( $\mathbf{Q}$ ), a systemically treatable tumour ( $(\mathbf{O})$, and a tumour undiagnosed before death $(\Delta)$. There are no significant differences between the curves (log rank $1.5(P=0.47)$. signs suggesting pulmonary disease. Table 1 shows data from history and physical examination ranged by primary tumour position.

Twenty four of the 52 patients who were later found to have a primary lung tumour, and four of the 20 patients without, showed symptoms or signs suggesting such a tumour. This resulted in a positive predictive value of history and physical examination of $85 \%$.

All patients had a chest radiograph; abnormalities were detected in 56. Primary lung tumour was diagnosed in 47 patients (positive predictive value $84 \%$ ).

Five patients, who later proved to have a primary lung tumour, had normal chest radiographs. In three of these, no additional diagnostic procedures were performed antemortem and the primary tumour was diagnosed at necropsy. In the two others, additional tomography or CT of the chest was performed on clinical grounds, disclosing abnormalities in both. The primary tumour was then confirmed by bronchoscopy. Tomography and CT of the chest were performed as part of the initial staging procedures in 39 patients. Mammography was performed in 13 of 26 women, disclosing abnormalities in four $(31 \%)$. Breast biopsy was performed in five patients (four with abnormalities on mammography and one with a breast carcinoma stage $\mathrm{T} 4$, without mammography), showing abnormalities in three $(60 \%)$.

The primary tumour was verified before death in 53 patients $(74 \%)$. Lung cancer was the most common (48), followed by breast cancer (three), colon carcinoma (one), and melanoma (one). In five of 19 patients whose underlying malignancies were not found before death, necropsy was performed, disclosing primary tumours in four.

Malignancy was histologically confirmed within two weeks. Figure 1 shows the cumulative percentage of diagnosed cases.

Figure 2 shows the survival curves of patients with systemically treatable, nonsystemically treatable, and undiagnosed tumours. The difference between the curves is not significant $(\log \operatorname{rank} 1.50(P=0.47))$, nor is the survival analysis in patients with confirmed versus unconfirmed primary tumour site $(\log \operatorname{rank} 0 \cdot 15(P=0 \cdot 69))$.

\section{Discussion}

This study focused on the diagnostic approach to patients with precocious brain metastases. The primary tumour was identified before death in 53 of the 72 patients $(74 \%)$ and at necropsy in four. The primary site remained unknown in 15 patients, a figure which corresponds with comparable studies, in which the underlying malignancy could not be detected in a substantial percentage of the patients, even after necropsy. ${ }^{1-35-8}$

Most of the patients with an identified underlying malignancy had a primary lung cancer (52 of 57). This cancer also ranked first in other studies (table 2),,$^{1-36-8}$ although le Cesne et al found gastrointestinal tumours to be the most frequent. ${ }^{5}$ 
Table 2 Comparison with the literature on position of primary tumour

\begin{tabular}{|c|c|c|c|c|c|c|c|c|}
\hline & \multicolumn{8}{|l|}{ Authors } \\
\hline & le Cesne et al & Chee and Byrnes ${ }^{1}$ & $\begin{array}{l}\text { le Chevalier } \\
\text { et al }\end{array}$ & Debevec ${ }^{6}$ & $\begin{array}{l}\text { Dhopesh' } \\
\text { and Yagnik }\end{array}$ & $\begin{array}{l}\text { Hamann } \\
\text { et al }\end{array}$ & Merchut ${ }^{3}$ & $\begin{array}{l}\text { Present } \\
\text { study }\end{array}$ \\
\hline $\begin{array}{l}\text { Year } \\
\text { Period } \\
\text { Patients } \\
\text { Organ (\%): }\end{array}$ & $\begin{array}{l}1991 \\
1959-80 \\
302\end{array}$ & $\begin{array}{l}1988 \\
1973-84 \\
51\end{array}$ & $\begin{array}{l}1985 \\
1959-79 \\
120\end{array}$ & $\begin{array}{l}1990 \\
1973-87 \\
75\end{array}$ & $\begin{array}{c}1985 \\
? \\
26\end{array}$ & $\begin{array}{l}1993 \\
1975-89 \\
122\end{array}$ & $\begin{array}{l}1989 \\
1977-87 \\
56\end{array}$ & $\begin{array}{l}1995 \\
1987-93 \\
72\end{array}$ \\
\hline $\begin{array}{l}\text { Lung } \\
\text { Digestive tract } \\
\text { Breast } \\
\text { Melanoma } \\
\text { Urinary tract } \\
\text { Other } \\
\text { Unknown }\end{array}$ & $\begin{array}{r}17 \\
38 \\
0 \\
-\quad \\
16 \\
13 \\
16\end{array}$ & $\begin{array}{r}41 \\
10 \\
10 \\
-\quad \\
8 \\
0 \\
31\end{array}$ & $\begin{array}{r}23 \\
11 \\
3 \\
4 \\
8 \\
8 \\
3 \\
48\end{array}$ & $\begin{array}{r}53 \\
1 \\
3 \\
3 \\
1 \\
1 \\
37\end{array}$ & $\begin{array}{l}\frac{62}{-} \\
\frac{4}{35}\end{array}$ & $\begin{array}{r}48 \\
4 \\
1 \\
5 \\
1 \\
42\end{array}$ & $\begin{array}{r}68 \\
9 \\
2 \\
2 \\
2 \\
4 \\
16\end{array}$ & $\begin{array}{r}72 \\
1 \\
4 \\
1 \\
0 \\
0 \\
21\end{array}$ \\
\hline
\end{tabular}

In the Netherlands, lung cancer is the most often diagnosed primary tumour $(15 \%$ of diagnosed tumours). ${ }^{9}$ An estimated one third of all patients with brain metastases have a primary lung cancer. ${ }^{10}$ Lung tumours thus seem to be relatively overrepresented in our patients.

In all patients with brain metastases, breast cancer is usually the second most common primary tumour. ${ }^{10}$ This does not apply, however, to studies of patients with precocious brain metastases. ${ }^{1-35-8}$ The premise of these studies is that breast cancer spreads less readily to the brain than lung cancer. Yet, breast cancer was the second most common primary tumour in our study.

Given the high frequency of primary lung tumours, the search for the primary tumour in patients with precocious brain metastases should be mainly directed towards the lung. In cases with a positive history or physical examination for primary lung tumour but a negative chest radiograph, a CT of the chest is indicated. Chest radiographs and CT proved very useful in detecting primary lung tumours, partly explained by the high percentage of these tumours. Other ancillary diagnostic procedures should be carried out if the patient's initial history or physical examination offer serious grounds for suspicion. Even with pathological confirmation of metastatic dis- ease, identification of the primary tumour is not likely if chest radiology is negative.

In terms of duration of survival, the prognosis of patients with a confirmed primary tumour site was not better. Even patients with a systemically treatable primary tumour did not show longer survival times. Routine diagnostic protocols are unnecessarily aggravating for the patient, time consuming, and not cost effective.

1 Chee CP, Byrnes DP. Cases of brain metastasis presenting as the first sign of systemic cancer. Singapore Med $\mathcal{F}$ 1988;29:252-6.

2 le Chevalier T, Smith FP, Caille P, Constans JP, Rouesse JG. Sites of primary malignancies in patients presenting with cerebral metastases. Cancer 1985;56:880-2.

3 Merchut MP. Brain metastases from undiagnosed systemic neoplasms. Arch Intern Med 1989;149:1076-80.

4 Lassouw GMJ, Twijnstra A, Schouten LJ, van de Pol M. The neuro-oncology register. Neuroepidemiology 1992;11: 261-6.

5 le Cesne A, le Chevalier T, Caille $P$, et al. Métastases de cancers à point de départ inconnu; Enseignement de 302 autopsies. La Presse Médicale 1991;20:1369-73.

6 Debevec M. Management of patients with brain metastases of unknown origin. Neoplasma 1990;37:601-6.

7 Dhopesh VP, Yagnik PM. Brain metastasis: analysis of patients without known cancer. South Med $\mathcal{f} 1985 ; 78$ : 171-2.

8 Hamann G, Meier T, Schimrigk K. Hirnmetastasen als erstmanifestation einer tumorerkrankung. Nervenarzt 1993;64:104-7.

9 Visser O, Coebergh JWW, Schouten LJ, ed. Incidence of cancer in the Netherlands 1991. Third report of the Netherlands cancer registry. Utrecht: SIG Health Care Information, 1994:98.

10 Posner JB, Chernik NL. Intracranial metastases from systemic cancer. Adv Neurol 1978;19:579-92. 